\title{
On strong uniform distribution, II. The infinite-dimensional case
}

\author{
by
}

\author{
Y. LACROIX (Brest)
}

We construct infinite-dimensional chains that are $L^{1}$ good for almost sure convergence, which settles a question raised in this journal [N]. We give some conditions for a coprime generated chain to be bad for $L^{2}$ or $L^{\infty}$, using the entropy method. It follows that such a chain with positive lower density is bad for $L^{\infty}$. There also exist such bad chains with zero density.

0. Introduction. A chain $\mathcal{C}$ is a multiplicative semigroup of the one of positive integers $\mathbb{N}$. We say a sequence $\mathbf{p}=\left(p_{k}\right)$ generates the chain $\mathcal{C}$ if $\mathcal{C}=\left\{\prod_{k=1}^{J} p_{k}^{\alpha_{k}}: \alpha_{k} \geq 0, J \geq 1\right\}$. A chain is of finite dimension (abbreviated FD) if there exists a finite sequence generating it; else, it is infinite-dimensional (abbreviated ID).

For example, $\mathbb{N}$ is ID and generated by the set $\mathcal{P}$ of all primes. Disproving Khinchin's strong uniform distribution conjecture, Marstrand $[\mathrm{M}]$ proved that there exists an open subset $V$ of the torus $\mathbb{T}$ such that the averages

$$
\frac{1}{N} \sum_{n=1}^{N} \mathbf{1}_{V}(n x \bmod 1)
$$

fail to converge a.e. with respect to Lebesgue measure $\lambda$.

He also proved that if $\mathcal{C}$ is finitely generated by prime numbers and $\left(n_{k}\right)$ denotes the increasing sequence such that $\left\{n_{k}: k \geq 1\right\}=\mathcal{C}$, then the averages

$$
\frac{1}{K} \sum_{k=1}^{K} f\left(n_{k} x \bmod 1\right)
$$

converge a.e. to $\int_{\mathbb{T}} f d \lambda$ for $f \in L^{\infty}(\mathbb{T}, \lambda)$.

1991 Mathematics Subject Classification: Primary 11K, 28D.

Key words and phrases: dimension, chains, almost sure convergence, universally good, density. 
Later, R. Nair $[\mathrm{N}]$ observed that Marstrand's preceding result could be extended to $f \in L^{1}(\mathbb{T}, \lambda)$, making use of the multidimensional ergodic theorem $([\mathrm{Be}],[\mathrm{K}])$. He raised the question of the existence of ID chains such that the averages in (1) converge a.e. for any $f \in L^{1}(\mathbb{T}, \lambda)$.

In this note we give an affirmative answer to R. Nair's question, together with some hints towards the use of the entropy method [Bo] for proving some chain is bad.

The problem of a.s. convergence of averages (1) developed parallel to the Riemann sum problem, which is that of a.s. convergence of the averages

$$
\frac{1}{n_{k}} \sum_{i=1}^{n_{k}} f\left(x+\frac{i}{n_{k}} \bmod 1\right) .
$$

For the latter, after $\mathrm{W}$. Rudin $[\mathrm{R}]$ showed that for any ID chain $\mathcal{C}=\left\{n_{k}\right.$ : $k \geq 1\}$ the convergence fails to hold a.e. for some $f \in L^{\infty}(\mathbb{T}, \lambda)$, in [DP] and later $[\mathrm{BW}]$ it was proved that the optimal functional space of convergence was $L \log L^{d-1}$ if the chain is generated by $d$ primes for instance (see also $[\mathrm{J}],[\mathrm{B}]$, and [N1] for this problem). Hence our main result (Theorem 1) strengthens the difference between these two problems.

In the first section, we construct explicit examples of ID chains that are good for a.s. convergence for any $f \in L^{1}(\mathbb{T}, \lambda)$. We make a careful use of the Tempel'man Ergodic Theorem for actions of the amenable semigroup $\bigcup_{q} \mathbb{N}^{q}$ $[\mathrm{K}]$. This essentially relies on proving some "covering lemma" .

The second section completes our answer to Nair's question: we give in Theorem 2 some criteria for a chain to be bad for $L^{2}$ or $L^{\infty}$, using the entropy method due to J. Bourgain [Bo] (used also in [BW] for the Riemann sum problem). In particular, a chain with positive lower density is bad for $L^{\infty}$, and there exist such bad chains with zero density.

Our results are summarized by the following:

THEOREM 0. There exist increasing subsequences $\mathbf{p}=\left(p_{k}\right)$ of coprime integers such that a.s. convergence in (1) holds for any $f \in L^{1}(\mathbb{T}, \lambda)$ along $\mathcal{C}(\mathbf{p})$. Moreover, the a.s. limit is $\int_{\mathbb{T}} f d \lambda$.

If a coprime generated chain $\mathcal{C}(\mathbf{p})$ has positive lower density, i.e.

$$
d_{\star}(\mathcal{C}(\mathbf{p}))=\liminf _{N} \frac{\# \mathcal{C}(\mathbf{p}) \cap[1, N]}{N}>0,
$$

then a.s. convergence in (1) fails for some $f \in L^{\infty}(\mathbb{T}, \lambda)$.

There exists such a chain with

$$
d(\mathcal{C}(\mathbf{p}))=\lim _{N} \frac{\# \mathcal{C}(\mathbf{p}) \cap[1, N]}{N}=0 .
$$

The author would like to thank R. Nair for helpful discussions during his visit at the Brest Mathematics Department, February 1996. 


\section{Good ID chains}

1.1. Ergodic theoretic preliminaries. We shall make an essential use of the following abelian semigroup endowed with its counting measure (for a subset $T$, \#T denotes its cardinality):

$$
l_{0}(\mathbb{N}):=\left\{\left(\alpha_{i}\right)_{i \geq 1}: \alpha_{i} \in \mathbb{N}, \exists j, i>j \Rightarrow \alpha_{i}=0\right\} .
$$

Given $q$ an integer, we identify $\mathbb{N}^{q}$ with a subsemigroup of $\mathbb{N}^{q+1}$ and the latter with one of $l_{0}(\mathbb{N})$ via the following embedings:

$$
\begin{array}{ccccc}
\mathbb{N}^{q} & \hookrightarrow & \mathbb{N}^{q+1} & \hookrightarrow & l_{0}(\mathbb{N}) \\
\left(\alpha_{1}, \ldots, \alpha_{q}\right) & \mapsto & \left(\alpha_{1}, \ldots, \alpha_{q}, 0\right) & \mapsto & \left(\alpha_{1}, \ldots, \alpha_{q}, 0,0, \ldots\right) .
\end{array}
$$

Given a probability measure space $(X, \mathcal{B}, \mu)$ and a sequence $\left(T_{k}\right)_{k \geq 1}$ of commuting endomorphisms of $(X, \mathcal{B}, \mu)$ (i.e. each $T_{k}: X \rightarrow X$ is measurable, $T_{k} \mu=\mu$, and $\left.T_{k} \circ T_{k^{\prime}}=T_{k^{\prime}} \circ T_{k}\right)$ we define an action $\Gamma$ of $l_{0}(\mathbb{N})$ on $(X, \mathcal{B}, \mu)$ by

$$
\Gamma\left(\left(\alpha_{k}\right)\right):=\bigcirc_{k \geq 1} T_{k}^{\alpha_{k}}
$$

where $T_{k}^{0}$ is meant to be the identity map. Mainly in this paper the reader can consider that $T_{k} x=p_{k} x \bmod 1, X=\mathbb{T}$, and $\mu=\lambda$, the Lebesgue measure.

For any sequence $(T(n))$ of subsets of $l_{0}(\mathbb{N})$, consider the following multiple condition $(\mathrm{P})$ :

$$
\left\{\begin{array}{l}
(\mathrm{P} 1): 0<\# T(n)<\infty, \\
(\mathrm{P} 2): \forall \gamma \in l_{0}(\mathbb{N}), \lim _{n} \#((T(n)+\gamma) \triangle T(n)) / \# T(n)=0, \\
(\mathrm{P} 3): T(n) \subset T(n+1), n \geq 1, \\
\text { (P4) }: \exists K_{1}<\infty, \forall N, \lim _{n} \#(T(N)+T(n)) / \# T(n) \leq K_{1}, \\
\text { (P5) }: \exists K_{2}<\infty, \forall n, \#(T(n)-T(n)) / \# T(n) \leq K_{2},
\end{array}\right.
$$

where $T(n)-T(n):=\left\{\alpha \in l_{0}(\mathbb{N}): \exists \gamma \in T(n), \alpha+\gamma \in T(n)\right\}$.

Then if $(T(n))$ satisfies $(\mathrm{P})$, by the Tempel'man Ergodic Theorem [K, p. 224], for any $f \in L^{1}(\mu)$, the averages

$$
\frac{1}{\# T(n)} \sum_{\alpha \in T(n)} f \circ \Gamma(\alpha)(x)
$$

converge $\mu$-a.e.

In this case we say that $(T(n))$ is $L^{1} \operatorname{good}$ universal (for $l_{0}(\mathbb{N})$ actions). If $\mathcal{C}$ is an ID coprime generated chain, $T_{k} x=p_{k} x \bmod 1$, we shall see in 1.2 below that averages (1) taken along particular sequence $(T(n))$ of subsets of $l_{0}(\mathbb{N})$ coincide with those above, converge $\lambda$-a.e. (when $(\mathrm{P})$ holds), and the limit equals $\int_{\mathbb{T}} f d \lambda$ (because the action is ergodic $[\mathrm{K}]$ ).

Definition 1. If averages (1) converge a.s. for any $f \in L^{p}(\mathbb{T})$, we say that $\mathcal{C}$ is a good chain (for $L^{p}$ ); otherwise $\mathcal{C}$ is bad (for $L^{p}$ ). 
1.2. Condition $(\mathrm{P})$ for a pairwise coprime generated chain. In this subsection we reduce condition $(\mathrm{P})$ for chains. Let $p_{1}<p_{2}<\ldots$ be an increasing sequence of pairwise coprime integers, $\mathcal{C}$ denote the chain they generate, and $\left(n_{k}\right)$ denote the sequence constituted by the elements of $\mathcal{C}$ ordered by size. For given $q \geq 1$ and $n \in[1, \infty[$, we let

$$
\begin{aligned}
& T_{q}(n):=\left\{\left(\alpha_{1}, \ldots, \alpha_{q}\right) \in \mathbb{N}^{q}: \sum_{i=1}^{q} \alpha_{i} \log p_{i} \leq \log n\right\}, \\
& T(n):=\left\{\alpha=\left(\alpha_{i}\right) \in l_{0}(\mathbb{N}): \sum_{i \geq 1} \alpha_{i} \log p_{i} \leq \log n\right\} .
\end{aligned}
$$

If $q(n):=\max \left\{q: p_{q} \leq n\right\}$, then $T(n)=T_{q(n)}(n)$. Therefore, for given $q \geq 1$, both $\left(T_{q}(n)\right)$ and $(T(n))$ satisfy (P1), (P3), and (P5) with $K_{2}=1$, because $T(n)-T(n) \subset T(n)$.

Moreover, since $T(n) \subset T(N)+T(n)\left(\right.$ resp. $\left.T_{q}(n) \subset T_{q}(N)+T_{q}(n)\right)$, and since it is easily checked that

$$
\begin{gathered}
\#(T(N)+T(n)) \leq \# T(n)+\sum_{\gamma \in T(N)} \#((\gamma+T(n)) \backslash T(n)) \\
\text { (resp. \#(Tq } \left.\left.(N)+T_{q}(n)\right) \leq \# T_{q}(n)+\sum_{\gamma \in T_{q}(N)} \#\left(\left(\gamma+T_{q}(n)\right) \backslash T_{q}(n)\right)\right),
\end{gathered}
$$

we see that (P2) implies (P4) with $K_{1}=1$. Hence we deduce (cf. (2))

Lemma 1. The sequence $(T(n))$ (resp. $\left.\left(T_{q}(n)\right)\right)$ defined by $(3)$ is $L^{1}$ good universal for $l_{0}(\mathbb{N})$ actions whenever it satisfies $(\mathrm{P} 2)$.

Given $\gamma=\left(\gamma_{i}\right) \in l_{0}(\mathbb{N})$, we have (also with $T(n)$ replaced by $T_{q}(n)$ )

(4) $\#((T(n)+\gamma) \triangle T(n))=\#(T(n) \backslash(T(n)+\gamma))+\#((T(n)+\gamma) \backslash T(n))$.

An elementary computation $[\mathrm{M}]$ shows that

$$
\# T_{q}(n) \sim \frac{(\log n)^{q}}{q ! \prod_{i=1}^{q} \log p_{i}} .
$$

Therefore, by (5), for any $\gamma \in l_{0}(\mathbb{N})$,

$$
\lim _{n} \#(T(n) \backslash(T(n)+\gamma)) / \# T(n)=0,
$$

hence with (4), (P2) reduces to

$$
\begin{aligned}
\forall \gamma \in l_{0}(\mathbb{N}), \quad \lim _{n} \#((T(n)+\gamma) \backslash T(n)) / \# T(n)=0 \\
\quad\left(\operatorname{resp} . \lim _{n} \#\left(\left(T_{q}(n)+\gamma\right) \backslash T_{q}(n)\right) / \# T_{q}(n)=0\right) .
\end{aligned}
$$


Now we show how this reduction is already applicable to the proof of [N, Thm. 1]. In addition to (5) we also have in $[\mathrm{M}]$

$$
\# \partial_{\bar{q}}\left(T_{q}(x)\right) \sim_{x \rightarrow \infty} \frac{\log \left(p_{1}^{q} \ldots p_{q}^{q}\right)}{(q-1) ! \prod_{i=1}^{q} \log p_{i}}(\log x)^{q-1} .
$$

Hence with (2), (5), (6), Lemma 1, (P6), and the fact that

$$
\left(T_{q}(n)+\gamma\right) \backslash T_{q}(n) \subset T_{q}\left(n \prod_{i=1}^{q} p_{i}^{\gamma_{i}}\right) \backslash T_{q}(n),
$$

a somewhat simplified proof of [N, Thm. 1] follows:

Corollary 1 . The sequence $\left(T_{q}(n)\right)$ is $L^{1}$ good universal for $l_{0}(\mathbb{N})$ actions.

1.3. The inductive step for constructing an ID good chain. Now we know that (P6) is enough for an ID coprime generated chain to be good, the idea is to show that given $p_{1}<\ldots<p_{q}$, it is possible to choose $p_{q+1}>p_{q}$ such that a "small" increase occurs in the quotients (P6) uniformly in $\gamma$ belonging to some finite subset $\langle q\rangle$ of $l_{0}(\mathbb{N})$, where the union over $q$ of these subsets cover $l_{0}(\mathbb{N})$.

Actually, this is possible thanks to the equality $T(n)=T_{q(n)}(n)$ and to a careful use of estimates (5) and (6). We start by presenting the analysis of this "control" (Lemma 2 below).

We assume $q>1$ and that $p_{1}<\ldots<p_{q}$ are pairwise coprime. We also let $p_{q+1}>p_{q}$ denote an integer coprime to the previous $p_{k}$ 's, to be specified later on. We let

$$
\langle q\rangle:=\left\{\gamma=\left(\gamma_{i}\right) \in \mathbb{N}^{q}: \gamma_{i} \leq q, 1 \leq i \leq q\right\},
$$

and $\bar{q}:=(q, \ldots, q) \in \mathbb{N}^{q}$. We define $\left(\gamma \leq \gamma^{\prime}\right) \Leftrightarrow\left(\forall i, \gamma_{i} \leq \gamma_{i}^{\prime}\right)$. We also introduce (cf. (P6))

$$
\partial_{\gamma}\left(T_{q}(n)\right):=\left(T_{q}(n)+\gamma\right) \backslash T_{q}(n) .
$$

Then an easy observation leads to

$$
\gamma \leq \gamma^{\prime} \Rightarrow \partial_{\gamma}\left(T_{q}(n)\right) \subset \partial_{\gamma^{\prime}}\left(T_{q}(n)\right) .
$$

Given arbitrary $\varepsilon_{q}>0$, using (5) and (6), we show as in Corollary 1 that there exists an $N\left(\varepsilon_{q}\right)$ such that

$$
x \geq N\left(\varepsilon_{q}\right) \Rightarrow \forall \gamma \in\langle q\rangle, \# \partial_{\gamma}\left(T_{q}(x)\right) / \# T_{q}(x)<\varepsilon_{q} / 2 .
$$

From now on we select $p_{q+1} \geq N\left(\varepsilon_{q}\right)\left(N\left(\varepsilon_{q}\right)\right.$ is as in (7)). Then if $k \geq 1$ and $p_{q+1}^{k} \leq n<p_{q+1}^{k+1}$, we have

$$
T_{q+1}(n)=\sum_{i=0}^{k}\left(\mathbb{N}^{q} \times\{i\}\right) \cap T_{q+1}(n) \quad \text { (disjoint union). }
$$


Set $T_{q+1}(n, i):=\left(\mathbb{N}^{q} \times\{i\}\right) \cap T_{q+1}(n), 0 \leq i \leq k=\left[\log n / \log p_{q+1}\right]$. Then we observe that $(0 \leq i \leq k)$

$$
\left(\alpha_{1}, \ldots, \alpha_{q}, i\right) \in T_{q+1}(n, i) \Leftrightarrow\left(\alpha_{1}, \ldots, \alpha_{q}\right) \in T_{q}\left(n / p_{q+1}^{i}\right),
$$

and moreover, if $\gamma \in\langle q\rangle$, then

$$
\partial_{\gamma}\left(T_{q+1}(n)\right)=\sum_{i=0}^{k} \partial_{\gamma}\left(T_{q+1}(n, i)\right) \quad \text { (disjoint union) }
$$

where $\partial_{\gamma}\left(T_{q+1}(n, i)\right)=\left(T_{q+1}(n, i)+\gamma\right) \backslash T_{q+1}(n, i)$. Moreover,

$$
\left(\alpha_{1}, \ldots, \alpha_{q}, i\right)+\gamma \in \partial_{\gamma}\left(T_{q+1}(n)\right) \Leftrightarrow\left(\alpha_{1}, \ldots, \alpha_{q}\right)+\gamma \in \partial_{\gamma}\left(T_{q}\left(n / p_{q+1}^{i}\right)\right) .
$$

Hence

$$
\begin{aligned}
\# T_{q+1}(n) & =\sum_{i=0}^{k} \# T_{q}\left(n / p_{q+1}^{i}\right), \\
\# \partial_{\bar{q}}\left(T_{q+1}(n)\right) & =\sum_{i=0}^{k} \# \partial_{\bar{q}}\left(T_{q}\left(n / p_{q+1}^{i}\right)\right) .
\end{aligned}
$$

Thus for any $n, p_{q+1} \geq N\left(\varepsilon_{q}\right)$, if $k=\left[\log n / \log p_{q+1}\right]$, we have, using (7):

$k=0$ (i.e. $\left.N\left(\varepsilon_{q}\right) \leq n<p_{q+1}\right) \Rightarrow T_{q+1}(n)=T_{q}(n)$

$$
\Rightarrow \forall \gamma \in\langle q\rangle, \# \partial_{\gamma}\left(T_{q+1}(n)\right) / \# T_{q+1}(n)<\varepsilon_{q} / 2,
$$

and

$$
\begin{aligned}
k \neq 0 \Rightarrow & \forall \gamma \in\langle q\rangle \\
& \# \partial_{\gamma}\left(T_{q+1}(n)\right) / \# T_{q+1}(n) \leq \# \partial_{\bar{q}}\left(T_{q+1}(n)\right) / \# T_{q+1}(n) \\
& \leq \frac{\sum_{i=0}^{k-1} \# \partial_{\bar{q}}\left(T_{q}\left(n / p_{q+1}^{i}\right)\right)}{\sum_{i=0}^{k-1} \# T_{q}\left(n / p_{q+1}^{i}\right)}+\# \partial_{\bar{q}}\left(T_{q}\left(n / p_{q+1}^{k}\right)\right) / \# T_{q}\left(n / p_{q+1}^{k-1}\right) \\
& <\varepsilon_{q} / 2+A\left(p_{q+1}, n / p_{q+1}^{k}\right),
\end{aligned}
$$

where $A\left(p_{q+1}, x\right)=\# \partial_{\bar{q}}\left(T_{q}(x)\right) / \# T_{q}\left(p_{q+1} x\right)(x \geq 1)$.

By (5) and (6) there exist two positive constants $C_{1}, C_{2}$ (depending on $p_{1}, \ldots, p_{q}$ and $q$, but not on $\left.p_{q+1}\right)$ such that for any $x, y \geq 1$,

$$
\# \partial_{\bar{q}}\left(T_{q}(x)\right) \leq C_{1}(\log x)^{q-1}, \quad \# T_{q}(y) \geq C_{2}(\log y)^{q} .
$$

Hence, (8) implies that, with $C_{3}=C_{1} / C_{2}$, and for any $x \geq 1$,

$$
A\left(p_{q+1}, x\right) \leq C_{3} / \log p_{q+1} .
$$

Let us select both $p_{q+1} \geq N\left(\varepsilon_{q}\right)$ and $C_{3} / \log p_{q+1}<\varepsilon_{q} / 2$ (here $C_{3}$ is given and the condition requires $p_{q+1}$ to be large enough). Then by (9), we see that as soon as $n \geq N\left(\varepsilon_{q}\right)$, for any $\gamma \in\langle q\rangle$,

$$
\# \partial_{\gamma}\left(T_{q+1}(n)\right) / \# T_{q+1}(n)<\varepsilon_{q} \text {. }
$$


We have proved:

Lemma 2. Given $q>1$, arbitrary coprime $p_{1}<\ldots<p_{q}$, and arbitrary $\varepsilon_{q}>0$, there exists an integer $N\left(\varepsilon_{q}\right)$ and a $p_{q+1} \geq N\left(\varepsilon_{q}\right)$ which is coprime to the $p_{i}$ 's $(1 \leq i \leq q)$ such that for any $\gamma \in\langle q\rangle$, if $n \geq N\left(\varepsilon_{q}\right)$, then (10) holds.

1.4. The inductive construction of an ID good chain. We fix a sequence $\left(\varepsilon_{q}\right)_{q \geq 1}$ of positive real numbers tending to 0 . Next we select an arbitrary $p_{1}>0$. Then a repeated inductive use of Lemma 2 produces a sequence $p_{1}<\ldots<p_{q+1}<\ldots$ of pairwise coprime integers, and another sequence $N\left(\varepsilon_{1}\right) \leq \ldots \leq N\left(\varepsilon_{q}\right) \leq \ldots$ of integers (we can choose them increasing).

We then define, for each $n$, the set $T(n)$ as in Subsection 1.2. As before, $T(n)=T_{q(n)}(n)$, where $p_{q(n)} \leq n<p_{q(n)+1}$ : thus if $n>p_{2}$ and $q \leq q(n)-1$, then

$$
\gamma \in\langle q\rangle \Rightarrow \# \partial_{\gamma}\left(T_{q(n)}(n)\right) / \# T_{q(n)}(n)<\varepsilon_{q(n)-1} .
$$

Now we fix $\gamma \in l_{0}(\mathbb{N})$ and select $q \geq 2$ such that $\gamma \in\langle q\rangle$. Then if $n_{0}$ satisfies $q\left(n_{0}\right)-1 \geq q$, we find that for any $n \geq n_{0}$,

$$
\# \partial_{\gamma}(T(n)) / \# T(n)=\# \partial_{\gamma}\left(T_{q(n)}(n)\right) / \# T_{q(n)}(n)<\varepsilon_{q(n)-1},
$$

by our inductive construction using Lemma 2 . Since $\varepsilon_{q} \rightarrow 0$ and $q(n) \rightarrow \infty$, this proves (P6), hence (P2). By Lemma 1, we obtain:

THEOREM 1. Let $p_{1}<p_{2}<\ldots$ be the sequence of pairwise coprime integers constructed above, and $\mathcal{C}$ the $I D$ chain it generates. Then $\mathcal{C}$ is good for $L^{1}$, and the almost sure limit in (1) equals $\int_{\mathbb{T}} f d \lambda$.

2. Bad ID chains. In this section we aim to present some material derived from [Bo], that may be useful in proving an ID chain is bad: the criteria are listed in Theorem 2, and Corollary 2 and Proposition 1 give some straightforward application of them, which is used in Theorem 3 to prove existence of an ID bad chain with zero density.

We consider an ID coprime generated chain $\mathcal{C}, \mathcal{C}=\mathcal{C}(\mathbf{p})$, and $\mathbf{p}=\left(p_{k}\right)$. As before we let $\left(n_{k}\right)$ be increasing and such that $\mathcal{C}=\left\{n_{k}: k \geq 1\right\}$. For $f \in L^{2}(\mathbb{T}, \lambda)$, we let

$$
S_{K} f(x):=\frac{1}{K} \sum_{k=1}^{K} f\left(n_{k} x\right), \quad x \in \mathbb{T} .
$$

The entropy method of Bourgain is based on the following facts [Bo, Prop. 1, 2]: if $\left(S_{K} f(x)\right)$ converges $\lambda$-a.e. for all $f \in L^{\infty}(\mathbb{T}, \lambda)$ (resp. $f \in$ $\left.L^{2}(\mathbb{T}, \lambda)\right)$ with $\|f\|_{2} \leq 1$, then for any $\delta>0$, there are uniform entropy estimates for such $f$ 's, i.e. there exists $C(\delta)<\infty$ (resp. there exists a $C>0$ ) such that

$$
N(f, \delta) \leq C(\delta) \quad(\operatorname{resp} . \delta \sqrt{N(f, \delta)}<C)
$$


$\left(N(f, \delta)\right.$ refers to the delta entropy number of the sequence $\left(S_{K} f\right)$ in Hilbert space $L^{2}(\mathbb{T}, \lambda)$, which is the minimal number of $L^{2}$ balls of radius $\delta$ centered at $S_{K} f$ 's needed to cover the set $\left\{S_{K} f: K \geq 1\right\}$ ).

This was used in [Bo] to recover Rudin's as well as Marstrand's results. We shall adapt Bourgain's approach to the latter so as to fit it to the ID chain case (notations are as in the previous sections).

For $q, k, c, T \geq 1$ and $0 \leq j<k$, we let (cf. (3))

$$
\begin{gathered}
A(j, c):=T\left(p_{1}^{(j+1) c}\right) \backslash T\left(p_{1}^{j c}\right), \quad A_{q}(j, c):=T_{q}\left(p_{1}^{(j+1) c}\right) \backslash T_{q}\left(p_{1}^{j c}\right), \\
A_{q}(T, j, c):=T_{q}\left(p_{1}^{T+(j+1) c}\right) \backslash T_{q}\left(p_{1}^{T+j c}\right) .
\end{gathered}
$$

For $B \subset l_{0}(\mathbb{N})$ finite and $f \in L^{2}(\mathbb{T}, \lambda)$, we let (cf. (2))

$$
S_{B} f(x):=\frac{1}{\# B} \sum_{\alpha \in B} f \circ \Gamma(\alpha)(x)
$$

$\left(\right.$ recall $\left.\Gamma(\alpha)(x)=\prod_{i \geq 0} p_{i}^{\alpha_{i}} x \bmod 1\right)$. And for given $c, k \geq 1$, we let

$$
q:=q(c, k)=q\left(p_{1}^{k c}\right) \quad\left(\text { recall } p_{q(n)} \leq n<p_{q(n)+1}\right)
$$

and define for given $T \geq 1(e(y):=\exp (2 i \pi y))$

$$
f^{(j)}=\sqrt{\# A_{q}(T, j, c)} S_{A_{q}(T, j, c)} e(x), \quad 0 \leq j<k .
$$

Then obviously

$$
\left\|f^{(j)}\right\|_{2}=1 \quad \text { and } \quad f^{(j)} \perp_{L^{2}} f^{\left(j^{\prime}\right)} \quad \text { if } 0 \leq j \neq j^{\prime}<k .
$$

Also, since $q=q\left(p_{1}^{k c}\right)$,

$w \in A(j, c)$ and $n \in A_{q}(T, 0, c) \Rightarrow w+n \in A_{q}(T, j, c) \cup A_{q}(T, j+1, c)$.

Set

$$
f:=f^{(0)} .
$$

Then, since $\# A_{q}(T, j, c) \leq \# A_{q}(T, j+1, c)$ (cf. (12)), we easily deduce that

$$
\left\langle S_{A(j, c)} f, f^{(j)}+f^{(j+1)}\right\rangle_{L^{2}} \geq \sqrt{\frac{\# A_{q}(T, 0, c)}{\# A_{q}(T, j+1, c)}} \geq \sqrt{\frac{\# A_{q}(T, 0, c)}{\# A_{q}(T, k-1, c)}} .
$$

Hence, letting $B(j, c)=A(0, c) \cup \ldots \cup A(j, c)\left(=T\left(p_{1}^{(j+1) c}\right)\right), 0 \leq j<k-1$, we get by positivity of the summands

$$
\begin{aligned}
& \left\langle S_{B(j, c)} f, \frac{f^{(j)}+f^{(j+1)}}{\sqrt{2}}\right\rangle_{L^{2}} \\
& \quad \geq \frac{\# A(j, c)}{\# B(j, c)} \sqrt{\frac{\# A_{q}(T, 0, c)}{2 \# A_{q}(T, j+1, c)}} \geq \frac{\# A(j, c)}{\# B(j, c)} \sqrt{\frac{\# A_{q}(T, 0, c)}{2 \# A_{q}(T, k-1, c)}} .
\end{aligned}
$$


Using (5) and (6), for given $k$ and $c$, we may find $T$ large enough so that

$$
\left\langle S_{B(j, c)} f, \frac{f^{(j)}+f^{(j+1)}}{\sqrt{2}}\right\rangle_{L^{2}} \geq \frac{1}{4} \cdot \frac{\# A(j, c)}{\# B(j, c)} .
$$

Define

$$
\varrho(k, c):=\varrho(c)=\min \left\{\frac{\# A(j+1, c)}{\# A(j, c)}: 0 \leq j<k-1\right\} \quad(\geq 1) .
$$

We introduce an orthonormal family

$$
\left(\phi^{(j)}\right):=\left(\frac{f^{(2 j)}+f^{(2 j+1)}}{\sqrt{2}}\right)_{0 \leq j<[k / 2]-1} .
$$

Moreover, if $j<l$, then $\left\langle S_{B(2 j, c)} f, \phi^{(l)}\right\rangle=0$. And using (13) and (14), for $T$ large enough, we get

$$
\left\langle S_{B(2 j, c)} f, \phi^{(j)}\right\rangle_{L^{2}} \geq \frac{1}{4} \cdot \frac{\varrho(c)^{2 j}}{1+\varrho(c)+\ldots+\varrho(c)^{2 j}}:=\beta_{2 j} .
$$

Define

$$
\begin{aligned}
& \beta_{k}:=\beta_{2([k / 2]-1)}, \\
& Q_{1}=\limsup _{k} \sup _{c \geq 1} \beta_{k} \sqrt{\log k}, \\
& Q_{2}=\limsup _{k} \sup _{c \geq 1} \beta_{k} .
\end{aligned}
$$

TheOREM 2. Let $\mathcal{C}$ be a coprime generated chain. If $Q_{1}=\infty$, then $\mathcal{C}$ is bad for some $f \in L^{2}(\mathbb{T})$, and if $Q_{2}>0$, then it is bad for some $f \in L^{\infty}(\mathbb{T})$.

Proof. Take $k \geq 1$ arbitrary, and notice that the finite sequence $\left(\beta_{2 j}\right)_{0 \leq j<[k / 2]-1}$ of $(15)$ is positive decreasing.

Using the Cauchy-Schwarz inequality in $L^{2}(\mathbb{T})$ we see that for $0 \leq j \neq$ $l<[k / 2]-1$ and $T$ large enough, given $c \geq 1$, if $l<j$, then

$$
\beta_{k} \leq \beta_{2 j} \leq\left|\left\langle S_{B(2 l, c)} f-S_{B(2 j, c)} f, \phi^{(j)}\right\rangle\right| \leq\left\|S_{B(2 l, c)} f-S_{B(2 j, c)} f\right\|_{L^{2}} .
$$

Hence $N\left(f, \beta_{k}\right) \geq k /(4+d)$ for some constant $d>0$ (depending only on 4 ).

Now if we go back to Bourgain's criteria stated at the beginning of this section, we see that the $L^{2}$ case is contradicted if $Q_{1}=\infty$, while for $0<\delta<\lim \sup _{k} \sup _{c>1} \beta_{k}$ the $L^{\infty}$ uniform entropy estimate holds, hence $L^{\infty}$ convergence fails if $Q_{2}>0$.

2.1. Bad ID chains and density. Let $I \subset \mathbb{N}$. Its lower (resp. upper) density, denoted by $d_{\star}(I)$ (resp. $\left.d^{\star}(I)\right)$ is defined by

$$
\liminf _{N} \frac{\#[1, N] \cap I}{N} \quad\left(\text { resp. } \limsup _{N} \frac{\#[1, N] \cap I}{N}\right) .
$$

We say $I$ has a density $d(I)$ if $d_{\star}(I)=d^{\star}(I)=: d(I)$. 
Proposition 1. Let $\mathcal{C}$ be a coprime generated chain. If $d_{\star}(\mathcal{C})>0$, then $\mathcal{C}$ is bad for $L^{\infty}$.

Proof. Suppose that $\delta=d_{\star}(\mathcal{C})>0$; then it is easy to observe that for any $k \geq 4$, there exist arbitrarily large $c$ 's such that $\varrho(k, c) \geq \delta p_{1}^{c} / 3$. Hence the " $\sup _{c} \beta_{k}$ " in $Q_{1}$ or $Q_{2}$ is at least $1 / 2$. By Theorem 2 the chain is bad.

Corollary 2. Assume $\mathcal{C}$ is generated by an increasing sequence $\mathbf{p}=$ $\left(p_{k}\right)$ of primes and let $\left(p_{j}^{\prime}\right)$ be the complementary sequence of primes. If $\sum_{j} 1 / p_{j}^{\prime}<\infty$, then $d(\mathcal{C})$ exists and equals $\prod_{j}\left(1-1 / p_{j}^{\prime}\right)$. Hence $\mathcal{C}$ is bad for $L^{\infty}$.

Proof. Applying [T, §III.1, Exercice 3(c)] (the Davenport-Erdös theorem (1951)), since $\sum_{j} 1 / p_{j}^{\prime}<\infty$, if $\mathcal{M}=\mathbb{N} \backslash \mathcal{C}=\bigcup_{j} p_{j}^{\prime} \mathbb{N}$, then

$$
d(\mathcal{M})=1-d(\mathcal{C})=1-\prod_{j}\left(1-\frac{1}{p_{j}^{\prime}}\right)<1 .
$$

So $d(\mathcal{C})$ exists and is strictly positive. It remains to apply Proposition 1.

2.2. Bad ID chains with zero density. We let $\mathcal{P}$ be the set of primes, and $\left(r_{t}\right)$ be its increasing enumeration. Let $4 \leq k_{1}<k_{2}<\ldots$ and $0=\alpha_{0}<\alpha_{1}<$ $\alpha_{2}<\ldots$ go to infinity, the $k_{i}$ 's being integers. We shall construct sequences $\left(t_{i}\right),\left(e_{i}\right),\left(c_{i}\right),\left(T_{i}\right)$ and $\left(f_{i}\right)$ such that:

(i) $t_{1}=1, t_{i}+e_{i}<t_{i+1}$;

(ii) if $\mathbb{N}_{1}:=\bigcup_{i>1}\left\{t_{i}, \ldots, t_{i}+e_{i}-1\right\}$, and for each $i \geq 1, \mathcal{C}_{i}$ denotes the chain generated by $\left\{r_{t}: t \in \mathbb{N}_{1} \cap\left[1, t_{i}+e_{i}-1\right]\right\}$, then for $c=c_{i}$ and $T=T_{i}$ (cf. (15)) $\beta_{k_{i}} \geq 1 / 2$ for $f=f_{i} \in L^{\infty}(\lambda),\left\|f_{i}\right\|_{2} \leq 1$, referring to the FD chain $\mathcal{C}_{i}$ (computations are done at each step $i$ as at the beginning of Section 2);

(iii) for each $i \geq 0, \sum_{t \notin \mathbb{N}_{1}, t<t_{i}} 1 / r_{t} \geq \alpha_{i}$;

(iv) $\mathcal{C}=\bigcup_{i \geq 1} \mathcal{C}_{i}$ is a chain, generated by $\left\{r_{t}: t \in \mathbb{N}_{1}\right\}$.

Induction. First step. As at the beginning of Section 2, and by Subsection 2.1 (Theorem 2), we may find $c_{1}$ and $T_{1} \geq 1$ such that if $e_{1}=$ $\# \mathcal{P} \cap\left[1, r_{1}^{k_{1} c_{1}}\right.$ [ then $\beta_{k_{1}} \geq 1 / 2$, where the computations refer to the FD chain generated by $\left\{r_{t}: 1 \leq t \leq 1+e_{1}-1\right\}$, and coincide with those that would be done for the chain $\mathbb{N}$. We let $f_{1}$ denote the $f^{(0)}$ from the beginning of Section 2, and $t_{1}=1$. Then $\mathbb{N}_{1} \cap\left[1, e_{1}\right]=\left[1, e_{1}\right]$ is constructed.

Inductive step. Assume that for some $i \geq 1$ the five finite sequences $\left(t_{j}\right)_{j \leq i},\left(e_{j}\right)_{j \leq i},\left(c_{j}\right)_{j \leq i},\left(T_{j}\right)_{j \leq i}$ and $\left(f_{j}\right)_{j \leq i}$ have been constructed and satisfy the desired conditions, and hence $\mathbb{N}_{1} \cap\left[1, t_{i}+e_{i}-1\right]$ is known. Then since $\mathcal{C}_{i}$ is generated by primes less than $t_{i}+e_{i}-1$, and $\sum_{t} 1 / r_{t}=\infty$, we can pick $t_{i+1}>t_{i}+e_{i}$ such that $\sum_{t_{i}+e_{i} \leq t<t_{i+1}} 1 / r_{t} \geq \alpha_{i}$. 
Since the chain $\widetilde{\mathcal{C}}_{i+1}$ generated by $\mathcal{P}_{i}:=\mathcal{P} \backslash\left\{r_{t}: t<t_{i+1}, t \notin \mathbb{N}_{1}\right\}$ has density 1 (positive), by the preceding subsection, we may find some $c_{i+1}$ such that if $e_{i+1}=\# \mathcal{P}_{i} \cap\left[1, r_{1}^{k_{i+1} c_{i+1}}\right.$ [ then $\beta_{k_{i+1}} \geq 1 / 2$, where again the computations refer to the FD chain $\mathcal{C}_{i+1}$ generated by $\mathcal{P}_{i} \cap\left[1, t_{i+1}+e_{i+1}[\right.$, and coincide with those that would be done for the chain $\widetilde{\mathcal{C}}_{i+1}, f_{i+1}$ being the corresponding " $f^{(0)}$ ", and $T_{i+1}$ the corresponding $T$. Then we let

$$
\mathbb{N}_{1} \cap\left[1, t_{i+1}+e_{i+1}-1\right]=\left(\mathbb{N}_{1} \cap\left[1, t_{i}+e_{i}-1\right]\right) \cup\left[t_{i+1}, t_{i+1}+e_{i+1}-1\right] .
$$

End of induction. From the construction it follows that $\mathcal{C}=\bigcup_{i} \mathcal{C}_{i}$ is a chain generated by $\left\{r_{t}: t \in \mathbb{N}_{1}\right\}$, and has the following two properties:

(a) $d(\mathcal{C})=0$ : indeed, since $\lim _{i} \alpha_{i} \leq \sum_{t \notin \mathbb{N}_{1}} 1 / r_{t}=\infty$, from [T, Exercice $6(\mathrm{e}), \mathrm{p} .281$ ] it follows that

$$
1 \geq d_{\star}(\mathbb{N} \backslash \mathcal{C})=1-\prod_{t \notin \mathbb{N}_{1}}\left(1-\frac{1}{r_{t}}\right)=1,
$$

hence by the identity

$$
d^{\star}(\mathcal{C})=\limsup _{N} \frac{\#(\mathcal{C} \cap[1, N])}{N}=1-d_{\star}(\mathbb{N} \backslash \mathcal{C})
$$

we deduce that $0=d^{\star}(\mathcal{C})=d(\mathcal{C})$.

(b) For each $i \geq 1$ we can find an $f \in L^{\infty}(\mathbb{T})\left(f=f_{i}\right)$, a $c \geq 1\left(c=c_{i}\right)$, and a $T \geq 1\left(T=T_{i}\right)$ such that $\beta_{k_{i}} \geq 1 / 2$, computed as in (15).

Hence the constructed ID chain $\mathcal{C}$ is such that $Q_{1}=\infty$ and $Q_{2}>0$, and we therefore deduce from Theorem 2 the following

Theorem 3. The chain $\mathcal{C}$ is bad for $L^{\infty}$ and satisfies $d_{\star}(\mathcal{C})=0$.

\section{References}

[B] R. C. Baker, Riemann sums and Lebesgue integrals, Quart. J. Math. Oxford Ser. (2) 27 (1976), 191-198.

[Be] T. Bewley, Extension of the Birkhoff and von Neumann ergodic theorems to semigroup actions, Ann. Inst. H. Poincaré Sect. B 7 (1971), 248-260.

[Bo] J. Bourgain, Almost sure convergence and bounded entropy, Israel J. Math. 63 (1988), 79-97.

[BW] Y. Bugeaud and M. Weber, Examples and counterexamples for Riemann sums, preprint, I.R.M.A., Strasbourg, 1996.

[DP] L. E. Dubins and J. Pitman, A pointwise ergodic theorem for the group of rational rotations, Trans. Amer. Math. Soc. 251 (1979), 299-308.

[J] B. Jessen, On the approximation of Lebesgue integrals by Riemann sums, Ann. of Math. 35 (1934), 248-251.

[K] U. Krengel, Ergodic Theorems, de Gruyter Stud. Math. 6, de Gruyter, Berlin, 1985 . 
[M] J. M. Marstrand, On Khinchin's conjecture about strong uniform distribution, Proc. London Math. Soc. (3) 21 (1970), 540-556.

[N] R. Nair, On strong uniform distribution, Acta Arith. 56 (1990), 183-193.

[N1] - On Riemann sums and Lebesgue integrals, Monatsh. Math. 120 (1995), 49-54.

[R] W. Rudin, An arithmetic property of Riemann sums, Proc. Amer. Math. Soc. 15 (1964), 321-324.

[T] G. Tenenbaum, Introduction à la théorie analytique et probabiliste des nombres, Vol. 1, Société Mathématique de France, 1995.

Département de Mathématiques

Faculté des Sciences

Université de Bretagne Occidentale

6 Avenue V. Le Gorgeu

B.P. 809

29285 Brest Cedex, France

E-mail: lacroix@univ-brest.fr 